\title{
Oil recovery from fractured reservoirs using in situ and preformed particle gels in micromodel structures
}

\author{
Samira Heidari ${ }^{1} \cdot$ Maryam Ahmadi $^{1} \cdot$ Feridun Esmaeilzadeh $^{1} \cdot$ Dariush Mowla $^{1}$
}

Received: 12 October 2018 / Accepted: 16 February 2019 / Published online: 11 March 2019

(c) The Author(s) 2019

\begin{abstract}
Oil extraction often accompanied with relatively large amounts of water production causes several environmental and mechanical problems, particularly in oil-fractured reservoirs. One way to alleviate this problem is to use gels as a blockage agent. In this study, several micromodels with different geometries of fractures including a simple fracture, step fracture, fracture with variable mouth and fracture with a tiny crack were prepared. The amount of oil recovery was measured from these micromodels in the presence or absence of gel. In situ and preformed particle gels (PPGs) were injected into the micromodels to compare their ability in blocking fractures and increasing oil recovery. The $\mathrm{BaCl}_{2} \cdot 2 \mathrm{H}_{2} \mathrm{O}$ salt solution was used to swell PPGs. The amount of salt concentration was optimized so that PPGs had maximum ability to block fractures. Several PPG concentrations, 1000, 2000, and 3000 ppm, were examined to optimize the amount of PPGs, which have to be injected into the micromodels. Injection flow rate was optimized so that salt water could efficiently sweep the matrix structure. Results showed that both PPG and in situ gels have a considerable effect on decreasing water production and increasing oil extraction. However, PPGs were superior in comparison to in situ gels to increase oil recovery. It was found that increasing salt concentration decreases the swelling percentage of PPGs. The optimum amount of injection flow rate was found to be $0.1 \mathrm{~mL} / \mathrm{hr}$ for all the prepared micromodels. The optimum concentration of PPGs was different for each micromodel.
\end{abstract}

Keywords Preformed particle gel (PPG) $\cdot$ In situ gel $\cdot$ Water conformance $\cdot$ Micromodel $\cdot$ Oil recovery

\section{Introduction}

Unwanted water production is one of the major drawbacks occurring during extracting oil from fractured reservoirs (Wang and Seright 2006; Yu et al. 2017). In most cases, it increases by increasing extracted hydrocarbons from oil reservoirs (Chen et al. 2018; Izadmehr et al. 2018). The pressure of the reservoir reduces proportional to the produced volume of excess water. Water produced with hydrocarbon fluids contains salt, heavy metals, radioactive materials, and dissolved organic materials that have serious environmental detriments. It reduces the life of oil wells and increases the cost of corrosion, separation, refining, and water treatment. Therefore,

Feridun Esmaeilzadeh

esmaeil@shirazu.ac.ir

1 Department of Chemical and Petroleum Engineering, School of Chemical and Petroleum Engineering, Enhanced Oil and Gas Recovery Institute, Advanced Research Group for Gas Condensate Recovery, Shiraz University, Shiraz 7134851154, Iran there is a vital need to control excess water production. Generally, there are several methods to control unwanted water production and to improve well production called conformance control (Sun et al. 2019). The conformance control methods are based on several important criteria including (1) preventing damage to the hydrocarbon-producing reigns, (2) permeability reduction of water-producing areas and (3) preventing the permeability reduction of hydrocarbon-producing areas. Gel treatment is one of the most effective methods to overcome excess water production (Chauveteau et al. 2001, 2003; Durán-Valencia et al. 2014; Elsharafi and Bai 2012; Heidari et al. 2018; Sang et al. 2014).

In general, two main types of gels used for this purpose including in situ gels (gelant) and performed particle gels (PPGs). The main difference between them is the mechanism of gelation (Imqam et al. 2016). Many studies have been carried out to check gelant effectiveness in blocking unwanted water production (Brattekås et al. 2015; Ganguly et al. 2001; Gao and Technology 2016; McCool et al. 2009; Seright 2001; Sydansk et al. 2004; Wang and Seright 2006). Feng et al. (2003) successfully used microgels for water shutoff. Firozjaii et al. 
(2018) used a new type of polymer, copolymer Zetag $8187 \mathrm{G}$, for rheological study at high temperature, $185^{\circ} \mathrm{C}$, and salinity, $150,000 \mathrm{ppm}$, and concluded that it is considerable for polymer flooding in sandstone cores. Rousseau et al. (2005) performed sandpack experiments to examine the flow and transportation of microgels. PPGs could eliminate some gelant drawbacks including the difficulty of controlling gelatinization time, gelation uncertainty and diluting by forming water and minerals (Bai et al. 2007a, b; Chauveteau et al. 2001, 2004, 2003). Usually, most PPGs have acrylamide monomers in their structures. PPGs could swell to several times of their original sizes. Another important characteristic of PPGs is that they could be prepared on the surface and then injected into the reservoir. Therefore, PPG is less sensitive to physical and chemical conditions of the reservoir such as $\mathrm{pH}$, temperature, hydrogen sulfide, and shear rate. Heidari et al. (2018) synthesized an efficient copolymer of acrylamide and acrylic acid and determination of all the important parameters that can affect the swelling capacity of the synthesized PPGs including AAm/AA mole ratio, MBA mole percentage, types of salt solutions, time of swelling and $\mathrm{pH}$. Commercial PPGs are presented in different sizes. Seright (2001) demonstrated that gel could not penetrate into porous media after gelation. It approved that PPG could reduce water production without causing substantial damage. Bai and Zhang (2011) introduced a mathematical model. The model showed how PPGs extent in a porous media. They concluded that $\mathrm{pH}$ sensitive polymer gels are good candidates for water conformance in the fractured reservoirs. Seright (2003) showed that PPGs have better efficiency than in situ gels for water conformance. Zhang (2014) used a transparent fractured model to see the gel expansion in the open fractures. They studied the flow of water in the fractures filled by PPGs. They found that although PPGs could significantly reduce the permeability of the fractures, they could not completely block the fractures. Goudarzi et al. (2016) used a transparent fracture model to determine the distribution of microgels into the porous media. Bai et al. (2013) discussed their field and laboratory experiences about using PPGs and their benefits. They revealed that the resistance factor and fluid viscosity are vital factors affecting water conformance. Chancellor et al. (2016) examined the effect of temperature on the swelling percentage of PPGs.

Micromodels are used as the synthetic two-dimensional porous media in a small scale to see events occurring at different geometries of fractures in the scale of pores. Hitherto, many attempts have been made to use micromodels for studying water conformance in porous media. Gogoi et al. (2019) studied the flow behavior and enhanced oil recovery process in micromodels. Agi et al. (2018) introduced natural polymers used in enhancing oil recovery processes and the mechanism can affect their movement behavior in porous media. Bai et al. (2007b) examined the transportation mechanism of PPGs through porous media. It was demonstrated that PPG propagation through micromodels has six different patterns. Cost et al. (2000) defined three mechanisms of PPG movement into a micromodel including particles' deformation, shrinkage, and breakage. Wu and Bai (2008) introduced a mathematical model that revealed how PPGs expand in the porous medium. Imqam et al. (2015) suggested methods that minimize the PPG penetration into the matrix.

Despite all studies performed using PPGs for water conformance, there are still many ambiguities about the PPG performance in a fractured porous media. To the best of our knowledge, there is no report in the literature to study the PPG blockage in fractured micromodels with different geometries of fractures and to compare the performance of PPGs against in situ gels. To do so, PPG samples were first synthesized and swollen in $\mathrm{BaCl}_{2} \cdot 2 \mathrm{H}_{2} \mathrm{O}$ salt solutions based on the best physical conditions obtained in our previous study (Heidari et al. 2018). Then, several micromodels with different fractures were prepared based on Asmari oil reservoir geometry located in the south of Iran. Several experiments were performed to find the optimum amount of brine concentration, PPG concentration, and flow rate. Next, PPG samples and in situ gels were separately injected into the micromodels. Finally, oil recovery efficiency was measured to compare the PPG blockage with in situ gels conformance.

\section{Materials and instruments}

Acrylamide (AAm, > 99\%), acrylic acid (AA, > 99\%), polyethyleneimine (PEI, > 99\%), polyacrylamide (HPAM, $>99 \%$ ), $N, N^{\prime}$-methylenebisacrylamide (MBA, $>99 \%$ ) and potassium persulfate (KPS, > 99\%) were prepared from Sigma-Aldrich Chemical Company (Milwaukee, WI). Sodium chloride $(\mathrm{NaCl},>99.5 \%)$ was purchased from Kanto Chemical Company, Japan. Barium chloride dehydrate $\left(\mathrm{BaCl}_{2} \cdot 2 \mathrm{H}_{2} \mathrm{O},>99 \%\right)$ was prepared from Merck Company. Hydrofluoric acid (HF, $40 \mathrm{wt} \%$ ) was provided from Kimia Mavad Company, Iran. Distilled water was used throughout this study. The crude oil was prepared from Ahvaz oil reservoir with the density and viscosity of $0.88 \mathrm{~g} / \mathrm{cm}^{-3}$ and 15.20 $\mathrm{cp}$ at the temperature of $25.60{ }^{\circ} \mathrm{C}$, respectively. A digital balance (Sartorius balance, BP 210S, German) was exploited to measure the weight of samples. A syringe pump (NE-4000 Double Syringe pump, USA) was used to inject fluids into the micromodels. A digital microscopic camera (Dino-Lite Digital Microscope, Taiwan) was used to see fluid movement in the porous media. The CrawlDraw software (2015) was utilized to design the artificial patterns. The laser device (MUV model) was implemented to carve the artificial patterns on the glass surface. Shimadzu FT-IR-8300 instrument recorded FT-IR spectra of samples. The specimens were grounded with $\mathrm{KBr}$ and subsequently pressed into a pellet to become ready for the IR test. Samples' spectra were collected in the range of $4000-500 \mathrm{~cm}^{-1}$. The scanning electron microscope 
(SEM, VEGA3 TESCAN, at $20 \mathrm{kV}$ ) derived scanning electron micrographs. Prior to SEM observation, sputter coater (VEGA3 TESCAN) was used to sputter-coat PPG samples with gold using physical vapor deposition (PVD) technique.

\section{Experiments}

\section{Experimental setup}

Figure 1 shows the micromodel setup. As can be seen, it consists of a syringe pump (No. 1), which injected fluid into the micromodel with a distinct flow rate and pressure, a light source (No. 2), which provided background light for clearer filming, a fracture micromodel (No. 3), which was connected to the syringe pump, a camera (No. 4), which recorded fluid distribution changes during brine injection, a cylinder (No. 5), which stored the output fluids, two laptops (No. 6), which recorded data, and a pressure differential gauge (No.7), which indicated the pressure.

\section{Micromodel design and fabrication}

To prepare micromodels, a core plug was prepared from Asmari oil reservoir (see Fig. 2a). A thin section of the rock was then made (see Fig. 2b), and its SEM image was taken (see Fig. 2c). After that, the artificial pattern was designed using the Crawl Draw software. Different fracture patterns including a simple fracture, step fracture, fracture with variable mouth and fracture with a tiny crack were added to the artificial pattern. These ultimate patterns were drawn on the glass surfaces using the laser device. Hydrofluoric acid

Fig. 1 Micromodel set up
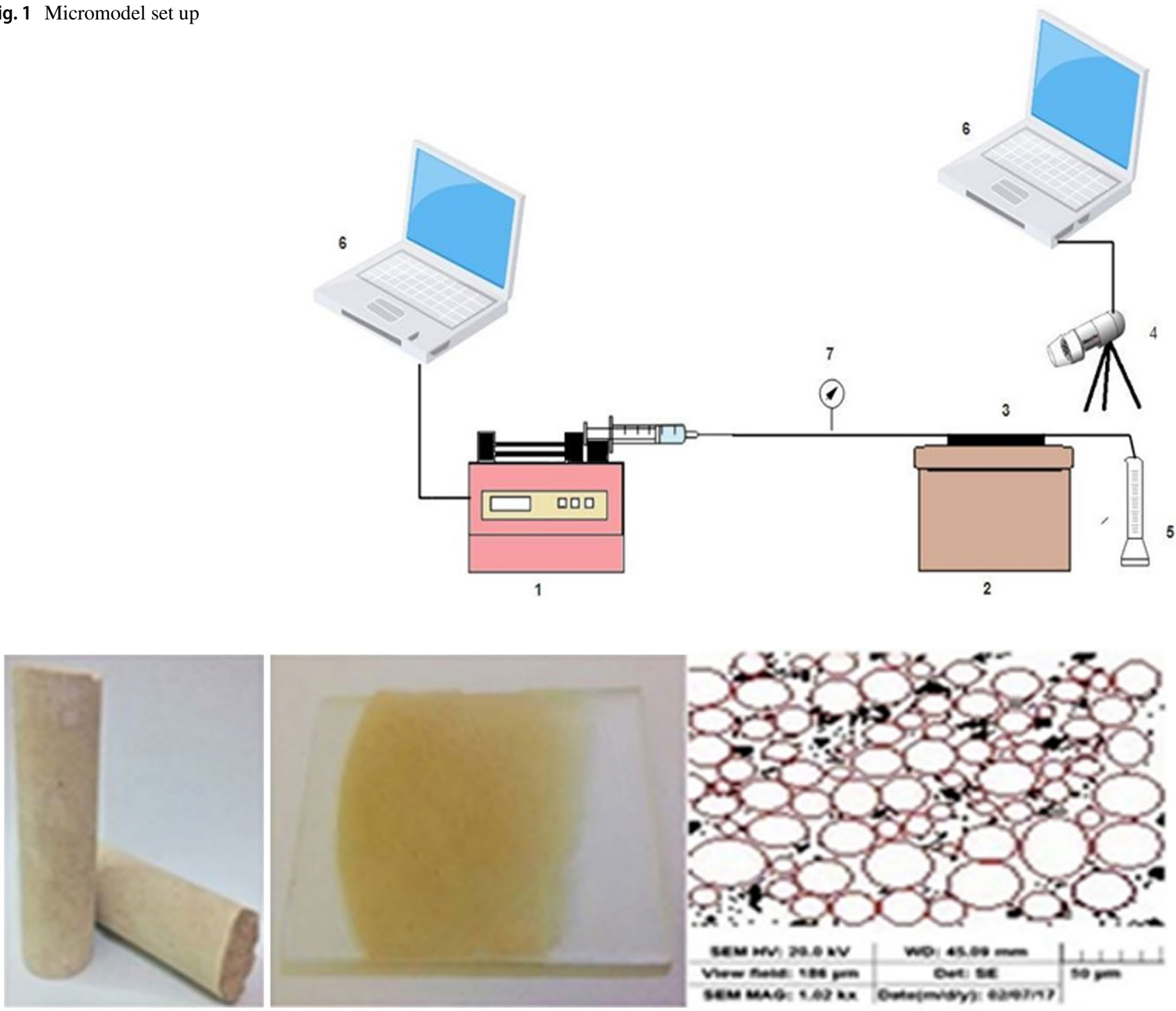

Fig. 2 Steps of preparing micromodels; $\mathbf{a}$ core sample, $\mathbf{b}$ thin section, $\mathbf{c}$ SEM image 

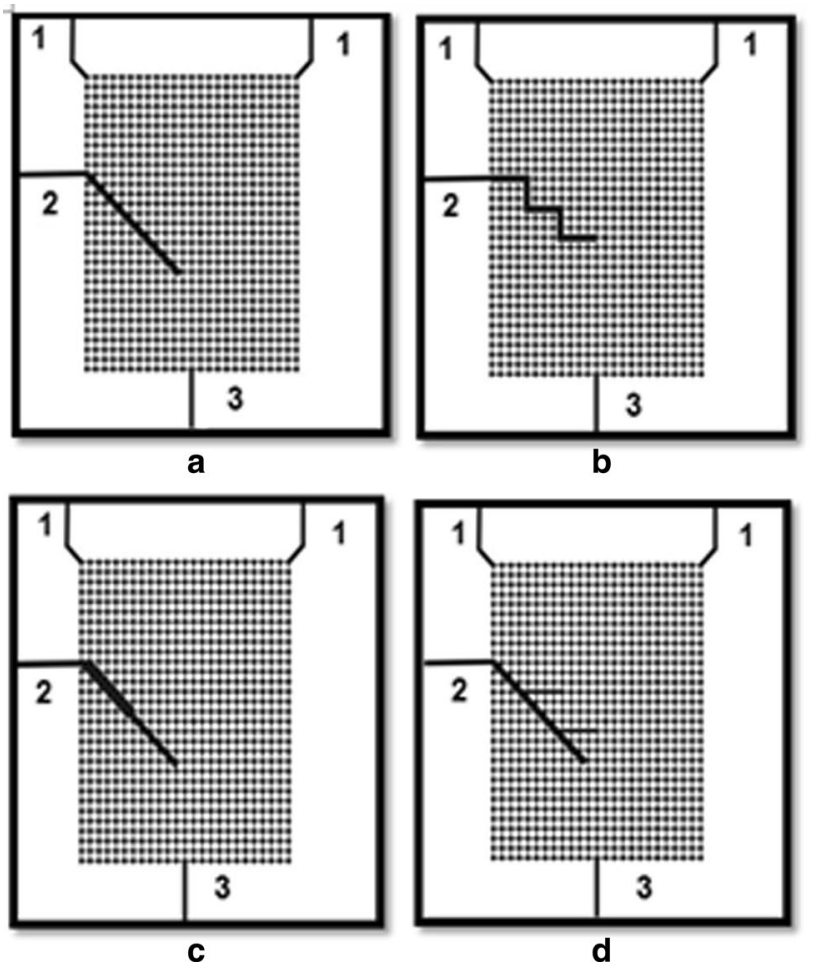

Fig. 3 Different micromodel patterns; $\mathbf{a}$ a simple fracture, $\mathbf{b}$ a stepped fracture, $\mathbf{c}$ a fracture with variable mouth, $\mathbf{d}$ a fracture with a tiny crack. *Numbers 1-3 indicate the water inlets, PPG inlet and the outlet, respectively

Table 1 The properties of the micromodels

\begin{tabular}{ll}
\hline Property & Value \\
\hline Dimension & $7.5 \mathrm{~cm} \times 10 \mathrm{~cm}$ \\
Pore diameter & $800 \mu \mathrm{m}$ \\
Throat diameter & $250 \mu \mathrm{m}$ \\
Fracture width & $1600 \mu \mathrm{m}$ \\
Crack width & $1200 \mu \mathrm{m}$ \\
Aspect ratio & 3.2 \\
\hline
\end{tabular}

was used to engrave the pattern on the glass surface. The designed glass surface was then stuck on a simple glass surface, which had the same material and dimension. Finally, the micromodel was put into the furnace (at 350, 550, 650, and $700{ }^{\circ} \mathrm{C}$ for $40,45,45$, and $45 \mathrm{~min}$, respectively) to achieve the desired two-dimensional space. Figure 3 shows the prepared micromodels. To provide a constant flow rate boundary condition, each micromodel had two water inlets connected to the syringe pump (No. 1) as shown in Fig. 3. PPG samples were injected into the micromodels using another entry (No. 2), and the outlet of the micromodel (No. 3) was connected to the atmosphere as shown in Fig. 3, which provided the constantpressure boundary condition. Table 1 shows the properties of the prepared micromodels. At the start of each experiment, the prepared micromodels were washed with toluene, ethanol, acetone, and distilled water, respectively.

\section{Procedure}

PPG samples composed of acrylamide and acrylic acid [poly (AAm-co-AA)] were synthesized based on the optimum conditions described in our previous study (Heidari et al. 2018). Then, they were powdered to particles with the size ranges from 3 to $10 \mu \mathrm{m}$. After that, PPGs were swollen in $\mathrm{BaCl}_{2} \cdot 2 \mathrm{H}_{2} \mathrm{O}$ salt solutions, and found to have the highest ability to swell PPGs (Heidari et al. 2018).

To prepare in situ gels, 7000-ppm polyacrylamide was added to the $50 \mathrm{~mL}$ distilled water existing in a $100 \mathrm{cc}$ beaker. The obtained solution was stirred for $2 \mathrm{~h}$ (until no polymer chains are visible in water). After that, the solution was kept under the atmospheric condition for 2 days without stirring to obtain a homogeneous solution. Next, $4000 \mathrm{ppm}$ polyethyleneimine and $10,000 \mathrm{ppm} \mathrm{NaCl}$ were added to the solution as a crosslinker agent and additive, respectively. The solution was stirred for $10 \mathrm{~min}$ to obtain a homogeneous gelant.

After preparing gel samples and micromodels, the experiments were performed in two sections: optimization and gel injection experiments.

\section{Optimization experiments}

The aim of this section was to determine the optimum concentration of the $\mathrm{BaCl}_{2} \cdot 2 \mathrm{H}_{2} \mathrm{O}$ salt solution used to swell PPGs and the optimum amount of PPGs that has to inject into each of the fractured micromodels.

To find the optimum concentration of salt solutions, the micromodels with a simple fracture, step fracture, fracture with variable mouth and fracture with a tiny crack were prepared as earlier described in the micromodel fabrication section. To do so, several $\mathrm{BaCl}_{2} \cdot 2 \mathrm{H}_{2} \mathrm{O}$ salt solutions, $1000,10,000$ and 100,000 ppm, were prepared. A certain amount of PPG, 2000 ppm, was swollen in each of the salt solutions. PPGs were rested in the salt solutions to swell up to $20 \mathrm{vol} \%$ of their maximum swelling capacities. Next, the swelled PPGs were quickly injected into each of the prepared micromodels. After that, the salt solutions were injected into the micromodels with different flow rates, $0.1,0.5,0.8$, and $1 \mathrm{~mL} / \mathrm{h}$ pressure drops were continuously recorded over the fractures. Certainly, there is a great pressure drop across the fractures if PPGs could perfectly swell in the presence of salt solutions particularly at a high-pressure salt solution. In this study, the worst conditions of the salt solutions were chosen to ensure that PPGs are able to increase oil recovery at the worst conditions.

To find the optimum amount of PPGs that has to be injected into the fractures, the micromodels were first prepared as described above. Different concentrations of PPG samples, 1000, 2000, and $3000 \mathrm{ppm}$, were then swollen in the 
$\mathrm{BaCl}_{2} \cdot 2 \mathrm{H}_{2} \mathrm{O}$ salt solution, which was prepared based on the worst conditions. PPGs were allowed to swell up to $20 \mathrm{vol} \%$ of their maximum swelling percentages. After that, the swelled PPGs were immediately injected into the micromodels. Next, the $\mathrm{BaCl}_{2} \cdot 2 \mathrm{H}_{2} \mathrm{O}$ salt solution prepared based on the worst conditions was injected into the micromodels with different flow rates, $0.1,0.5,0.8$, and $1 \mathrm{~mL} / \mathrm{hr}$ pressure drops were continuously recorded over the fractures. The obtained pressure drops disclosed the resistance of PPGs in the fractures.

\section{Micromodel experiments}

Experiments were started by measuring the real porosity of the micromodels. To do so, distilled water was first highlighted in blue color using methylene blue. The micromodels were then saturated with blue distilled water. The digital microscopic camera recorded images of the saturated micromodels. In the end, the real porosity was calculated using Matlab software (2017) based on surface dimensions and the average depth of dents, which depend on the immersion time of the glass in the hydrophilic acid.

To measure the absolute permeability of the micromodels, $\mathrm{BaCl}_{2} \cdot 2 \mathrm{H}_{2} \mathrm{O}$ salt solution prepared based on the worst conditions was injected into them. Then, pressure gradients between the inlet and outlet of the micromodels, and the injection flow rates were continually recorded. Absolute permeability was measured using Darcy law (Eq. 1).

$k=\frac{q \times \mu \times l}{A \times \Delta P}$

where $k$ is the absolute permeability (Wang and Seright 2006), $q$ is the flow rate $\left(\mathrm{cm}^{3} / \mathrm{s}\right), \mu$ is the fluid viscosity (cp), $l$ is the length $(\mathrm{cm}), A$ is the surface $\left(\mathrm{cm}^{2}\right)$ and $\Delta P$ is the pressure drop (atm.). As $l, A$, and $\mu$ are constant, therefore, $\Delta P$ chiefly changes by flow rate.

To perform the gel flooding process, the micromodels were first saturated with the $\mathrm{BaCl}_{2} \cdot 2 \mathrm{H}_{2} \mathrm{O}$ salt solution, prepared based on the worst conditions. The oil was then injected into the micromodels until no water was left out of the micromodels. At this time, there is only immobilized water in the micromodels. To obtain the amount of net oil recovery, the $\mathrm{BaCl}_{2} \cdot 2 \mathrm{H}_{2} \mathrm{O}$ salt solution was injected into the micromodels before injecting the gel into the fractures. Next, to measure the amount of improved oil recovery, the gel was first injected into the micromodels. In this study, both in situ and preformed particle gels were examined to compare their blockage ability. The micromodels filled with PPGs were rested for $3 \mathrm{~h}$ to obtain their maximum swelling percentage of PPGs. The micromodels filled with $0.2 \mathrm{PV}$ in situ gels were kept in the oven for $12 \mathrm{~h}$ at $100{ }^{\circ} \mathrm{C}$ to turn into the gel form. Next, the $\mathrm{BaCl}_{2} \cdot 2 \mathrm{H}_{2} \mathrm{O}$ salt solution was reinjected into the micromodels. The salt-water injection was continued until no oil was removed from the micromodels.

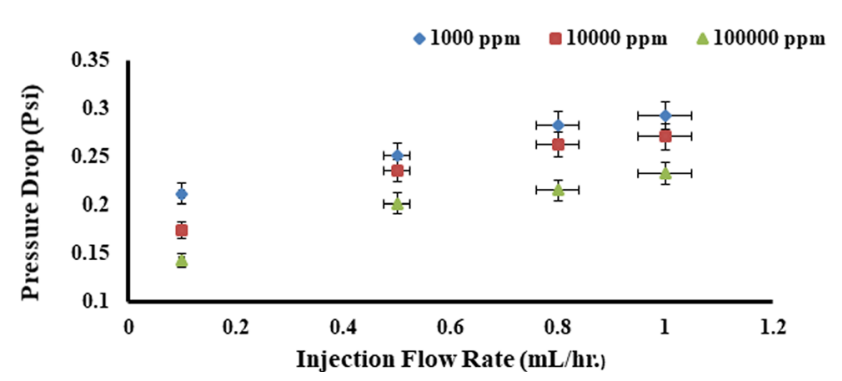

Fig. 4 Pressure drops versus injection flow rates for a simple fracture micromodel at different saline concentrations including 1000, 10,000, and $100,000 \mathrm{ppm}$ at $T=25^{\circ} \mathrm{C}$ and $P=0.846$ bar

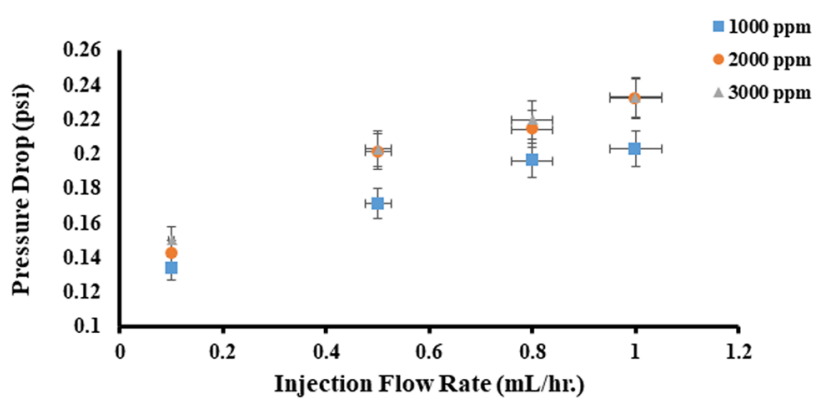

Fig. 5 Pressure drops versus injection flow rates for a simple fracture micromodel at different PPG concentrations including 1000, 2000, and $30,000 \mathrm{ppm}$ at $T=25^{\circ} \mathrm{C}$ and $P=0.846$ bar

After that, it was possible to calculate the remaining oil and blockage ability of gels.

\section{Results and discussion}

\section{Optimization of brine concentration}

Figure 4 shows the pressure drops obtained from each of the salt solution concentrations. As can be seen, the salt solution with the $100,000 \mathrm{ppm}$ of $\mathrm{BaCl}_{2} \cdot 2 \mathrm{H}_{2} \mathrm{O}$ had the minimum pressure drop across the fractures. It reveals that the pressure drop decreased by increasing salt solution concentration. It is due to the reduction of swelling percentage and therefore decreasing the blockage ability of PPGs by increasing salt concentration. In addition, pressure drop increased by increasing the flow rate. That is due to the straight relation between the injection flow rate and pressure drop.

\section{Optimization of PPG concentration}

Figure 5 illustrates the pressure drops obtained for each of PPG concentrations in the simple fracture micromodel. It is clear that pressure drop did not significantly change by increasing PPG concentration from 2000 to 3000 ppm.

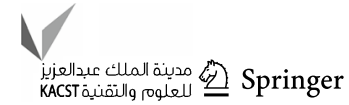


Therefore, the PPG concentration of $2000 \mathrm{ppm}$ was selected as the optimum PPG concentration in the simple fracture micromodel. The optimum PPG concentrations considered for the other micromodels with a step fracture, fracture with variable mouth and fracture with a tiny crack were found to be 1000,3000 , and 2000 ppm, respectively.

\section{Micromodel experiments}

\section{Real porosity and absolute permeability of the micromodels}

Obtained real porosity, absolute permeability and pore volume of the micromodels were given in Table 2. As it is clear, the fracture with variable mouth and the step fracture had the maximum and minimum amount of absolute permeability, respectively.

\section{Gel flooding process}

It was found that flow rate, fracture geometry and gel type could affect the amount of oil recovery. Hence, these factors were elaborated below in details.

Effect of flow rate Injection flow rate is one of the most important factors that affect the amount of oil recovery. Four

Table 2 Real porosity, absolute permeability, and pore volume of micromodels

\begin{tabular}{llll}
\hline Geometry & $\begin{array}{l}\text { Absolute } \\
\text { perme- } \\
\text { ability }\end{array}$ & Real porosity & Pore volume \\
\hline Simple fracture & 2.68 & 0.3892 & 0.6321 \\
$\begin{array}{l}\text { Fracture with a tiny crack } \\
\text { Fracture with variable }\end{array}$ & 2.86 & 0.3899 & 0.6332 \\
$\quad 3.23$ & 0.3894 & 0.6324 \\
$\quad$ mouth & 2.28 & 0.3898 & 0.6330 \\
\hline
\end{tabular}

Fig. 6 Water (blue)-oil (black) distributions appeared at different flow rates at $T=25^{\circ} \mathrm{C}$ and $P=0.846$ bar in a simple fracture micromodel a $0.1 \mathrm{~mL} / \mathrm{h}$. b $0.5 \mathrm{~mL} / \mathrm{h} . \mathbf{c} 1 \mathrm{~mL} / \mathrm{h}$ constant flow rates, $0.1,0.5,0.8$ and $1 \mathrm{~mL} / \mathrm{h}$, were injected into all the micromodels, respectively. Figure 6 shows water (blue)-oil (black) distributions appeared at different flow rates. By increasing the flow rate, the injected saline water flowed more through fractures with low resistance, and therefore less oil was recovered. It can be due to short flooding time for saline water to pass through the matrix structure. Based on the amount of oil recovery, flooding time and turbulence effect, the optimum flow rate was found to be $0.1 \mathrm{~mL} / \mathrm{h}$ for all the micromodels in the presence or absence of gel. Figure 7 exemplifies the amount of oil recovery obtained from a micromodel with a simple fracture in the absence of gel. Water injection is a key factor pushing oil throughout its path. However, at a certain pore volume of injection water, water finally flows through a path with the least resistance. After that, it will not sweep the remaining oil. Consequently, oil will not have any macroscopic movement. At this pore volume where water pushes the maximum amount of oil, the recovery factor (RF) is maximized and reaches a plateau.

Effect of gel type Figure 8 shows water (blue)-oil (black) distributions during saline water injection at the rate of $0.1 \mathrm{~mL} / \mathrm{h}$ for micromodels including in situ gels and

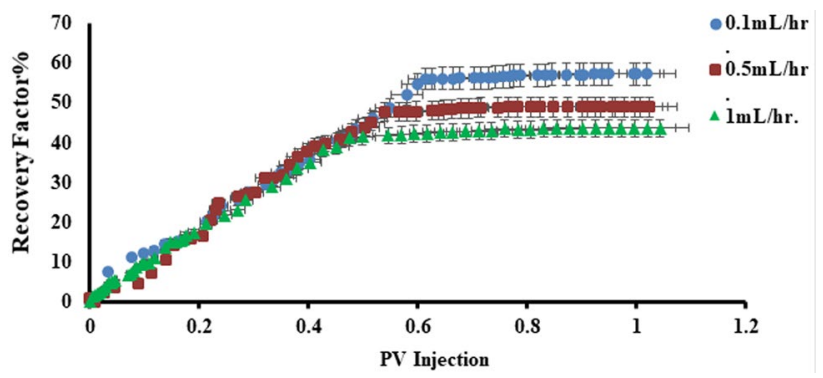

Fig. 7 The amount of oil recovery obtained from a simple fracure at different flow rates including $0.1,0.5$, and $1 \mathrm{~mL} / \mathrm{h}$ at $T=25{ }^{\circ} \mathrm{C}$ and $P=0.846$ bar
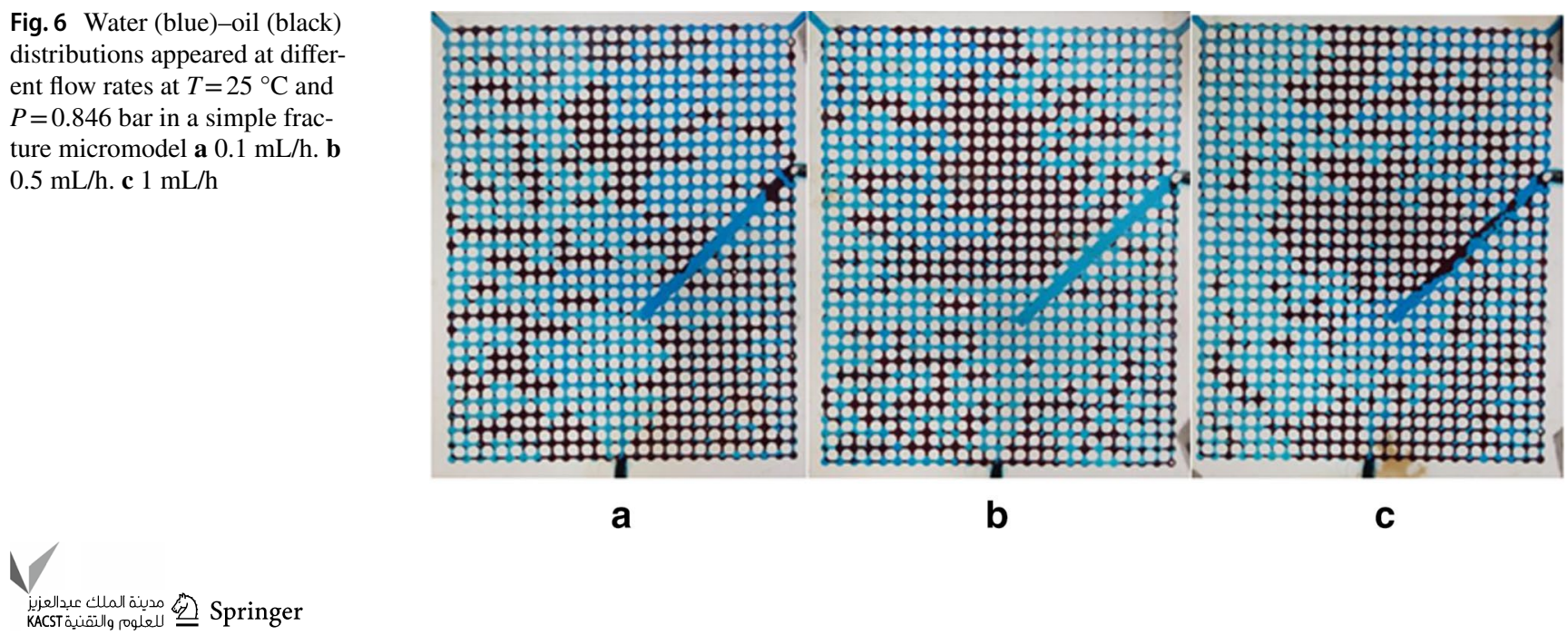
Fig. 8 Water (blue)-oil (black) distributions during salt water injection at the rate of $0.1 \mathrm{~mL} / \mathrm{hr}$ at $T=25{ }^{\circ} \mathrm{C}$ and $P=0.846$ bar. a The simple fracture micromodel with in situ gel, $\mathbf{b}$ the simple fracture micromodel with PPG

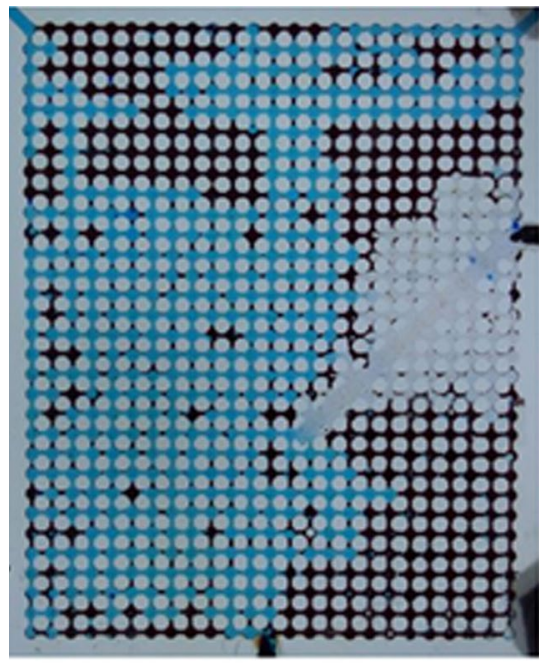

a

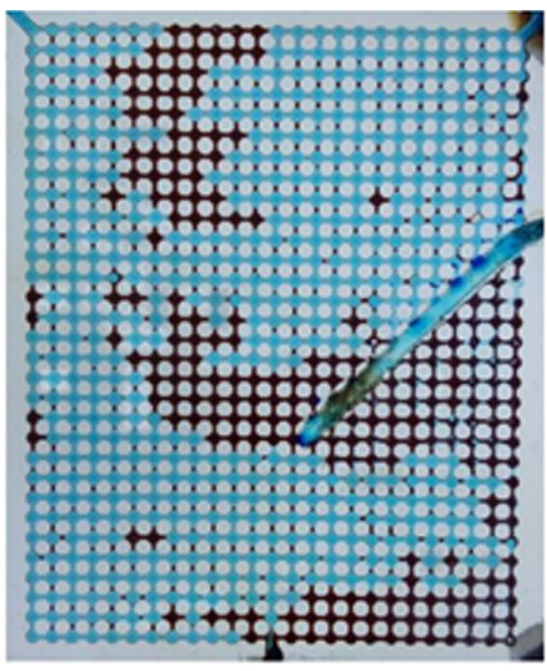

b

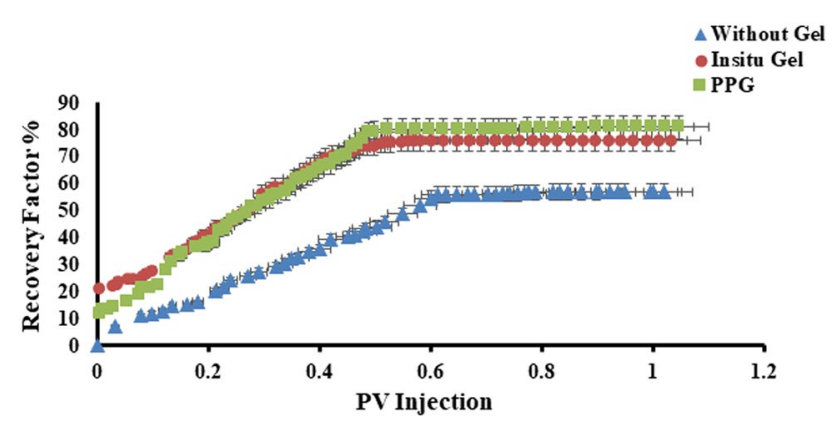

Fig. 9 The amount of oil recovery obtained from a simple fracure micromodel with in situ gel, with PPG and without gel at $T=25^{\circ} \mathrm{C}$ and $P=0.846$ bar

PPGs, respectively. As gels blocked fractures leading to increase the resistance of fractures; therefore, the injected salt water swept the matrix structure, and oil recovery was improved. Figure 9 confirmed that although the amount of salinity used in this study was not as high as that existing in a real formation water and all the experiments were conducted at ambient pressure and temperature, more oil recovery factor was obtained using PPGs. Although the difference in oil recovery factor for both gel types was not significant in this study (low pressure, temperature, and salinity), it is expected that more difference is achieved at the reservoir conditions (high pressure, temperature, and salinity) because PPGs can resist against many kinds of salt solutions with high concentrations and can withstand high pressures and temperatures, unlike in situ gels. Additionally, oil producers confirm that PPGs are more successful than in situ gels to control unwanted water production from oil or gas reservoirs (Bai et al. 1999, 2007b; Bai and Zhang 2011; Tongwa et al. 2013a, b).
Effect of fracture geometry Although oil recovery increased in all the micromodels after placing gels into the fractures, the maximum and minimum amount of oil recovery were obtained from the micromodels including the fracture with variable mouth and the step fracture, respectively. The fracture with variable mouth had high real permeability and low resistance against gel movement. Therefore, gels easily penetrated into it and blocked it. The tortuosity of the step fracture increased gel movement resistance and decreased oil recovery. In addition, the step fracture had the minimum amount of real permeability among all the prepared micromodels. Table 3 summarized the amount of oil recovery obtained from the micromodels.

\section{Conclusions}

In this study, several micromodels with different geometries of fractures including a simple fracture, step fracture, fracture with variable mouth and fracture with a tiny crack were prepared. PPGs were synthesized under optimum physical conditions and swelled at $\mathrm{BaCl}_{2} \cdot 2 \mathrm{H}_{2} \mathrm{O}$ salt solution. In situ gels were also synthesized. The maximum amount of oil recovery from the prepared micromodels was tried to achieve. To this end, the amount of $\mathrm{BaCl}_{2} \cdot 2 \mathrm{H}_{2} \mathrm{O}$ concentration, PPG concentration and flow rate were optimized. It was found that increasing salt concentration decreases the swelling percentage of PPGs and increasing the injection flow rate decreased oil recovery. Generally, it was found that both in situ gels and PPGs could block fractures and increase oil recovery. However, PPGs were more successful to resist against salt water and recovery of trapped oil. Altogether, these findings revealed that PPG treatment is an effective method to improve oil recovery from fractured reservoirs with different geometries of fractures.

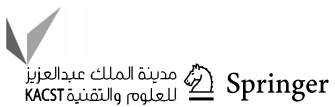


Table 3 The amount of oil recovery after injecting gel into the micromodels

\begin{tabular}{lll}
\hline Geometry & $\begin{array}{l}\text { Increased oil recovery after in situ gel } \\
\text { injection }\end{array}$ & $\begin{array}{l}\text { Increased oil } \\
\text { recovery after PPG } \\
\text { injection }\end{array}$ \\
\hline Injection flow rate $=0.1 \mathrm{~mL} / \mathrm{h}$ & & \\
Fracture with variable mouth & 21.6 & 24.66 \\
Fracture with a tiny crack & 20.09 & 23.77 \\
Simple fracture & 18.88 & 22.88 \\
Step fracture & 17.02 & 21.25 \\
\hline
\end{tabular}

Acknowledgements The authors are grateful to the Shiraz University and Enhanced Gas Condensate Recovery Research Group for supporting this research.

Open Access This article is distributed under the terms of the Creative Commons Attribution 4.0 International License (http://creativeco mmons.org/licenses/by/4.0/), which permits unrestricted use, distribution, and reproduction in any medium, provided you give appropriate credit to the original author(s) and the source, provide a link to the Creative Commons license, and indicate if changes were made.

\section{References}

Agi A, Junin R, Gbonhinbor J, Onyekonwu MJJoPE, Technology P (2018) Natural polymer flow behaviour in porous media for enhanced oil recovery applications: a review. 1-14

Bai B, Zhang H (2011) Preformed-particle-gel transport through open fractures and its effect on water flow. SPE Journal 16:388-400

Bai B, Li Y, Liu X (1999) New development of water shutoff and profile control in oilfields in China. Oil Drill Prod Technol 20:64-68

Bai B, Li L, Liu Y, Liu H, Wang Z, You C (2007a) Preformed particle gel for conformance control: factors affecting its properties and applications SPE reservoir. Eval Eng 10:415-422

Bai B, Liu Y, Coste J-P, Li L (2007b) Preformed particle gel for conformance control: transport mechanism through porous media. SPE Reserv Eval Eng 10:176-184

Bai B, Wei M, Liu Y (2013) Field and lab experience with a successful preformed particle gel conformance control technology. In: SPE production and operations symposium. Society of Petroleum Engineers

Brattekås B, Pedersen SG, Nistov HT, Haugen A, Graue A, Liang J-T, Seright R (2015) Washout of Cr (III)-acetate-HPAM gels from fractures: effect of gel state during placement. SPE Prod Oper 30:99-109

Chancellor C, Kirby C, Elsharafi M, Ok JT (2016) Control pH value for superabsorbent polymers (SAPS) solutions by using hydrochloride Acid (HCL). In: Proceedings of the 2016 Southwestern Petroleum Short Course Lubbock, Texas, USA, pp 20-21

Chauveteau G, Omari A, Tabary R, Renard M, Veerapen J, Rose J (2001) New size-controlled microgels for oil production. In: SPE international symposium on oilfield chemistry. Society of Petroleum Engineers

Chauveteau G, Tabary R, Le Bon C, Renard M, Feng Y, Omari A (2003) In-depth permeability control by adsorption of soft sizecontrolled microgels. In: SPE European formation damage conference. Society of Petroleum Engineers

Chauveteau G, Tabary R, Blin N, Renard M, Rousseau D, Faber R (2004) Disproportionate permeability reduction by soft preformed microgels. In: SPE/DOE symposium on improved oil recovery. Society of Petroleum Engineers
Chen H, Feng Q, Zhang X, Zhou W, Geng Y (2018) A prediction formula for ratio of injection-production control area in triangle well pattern. J Pet Explor Prod Technol 8:195-203

Coste J, Liu Y, Bai B (2000) Conformance control by preformed particle gel: Factors affecting its properties and application. In: Tulsa: SPE/DOE improved oil recovery symposium

Durán-Valencia C, Bai B, Reyes H, Fajardo-López R, Barragán-Aroche F, López-Ramírez S (2014) Development of enhanced nanocomposite preformed particle gels for conformance control in hightemperature and high-salinity oil reservoirs. Polym J 46:277

Elsharafi MO, Bai B (2012) Effect of weak preformed particle gel on unswept oil zones/areas during conformance control treatments. Ind Eng Chem Res 51:11547-11554

Feng Y, Tabary R, Renard M, Le Bon C, Omari A, Chauveteau G (2003) Characteristics of microgels designed for water shutoff and profile control. In: International symposium on oilfield chemistry. Society of Petroleum Engineers

Firozjaii AM, Zargar G, Kazemzadeh EJJoPE, Technology P (2018) An investigation into polymer flooding in high temperature and high salinity oil reservoir using acrylamide based cationic co-polymer: experimental and numerical simulation. 1-10

Ganguly S, Willhite G, Green D, McCool C (2001) The effect of fluid leakoff on gel placement and gel stability in fractures. Paper SPE

Gao CJJoPE, Technology P (2016) Application of a novel biopolymer to enhance oil recovery. 6:749-753

Gogoi S, Gogoi SBJJoPE, Technology P (2019) Review on microfluidic studies for EOR application:1-15

Goudarzi A, Alhuraishawy A, Taksaudom P, Sepehrnoori K, Bai B, Imqam A, Delshad M (2016) Experimental and simulation study of water shutoff in fractured systems using microgels. In: SPE Western Regional Meeting. Society of Petroleum Engineers

Heidari S, Esmaeilzadeh F, Mowla D, Ghasemi S (2018) Synthesis of an efficient copolymer of acrylamide and acrylic acid and determination of its swelling behavior. J Pet Explor Prod Technol 1-10

Imqam A, Bai B, Al Ramadan M, Wei M, Delshad M, Sepehrnoori K (2015) Preformed-particle-gel extrusion through open conduits during conformance-control treatments SPE J 20:1083-1093

Imqam A, Aldalfag A, Wang Y, Bai B (2016) Evaluation of preformed particle gels penetration into matrix for a conformance control treatment in partially open conduits. In: SPE annual technical conference and exhibition. Society of Petroleum Engineers

Izadmehr M, Daryasafar A, Bakhshi P, Tavakoli R, Ghayyem MA (2018) Determining influence of different factors on production optimization by developing production scenarios. J Pet Explor Prod Technol 8:505-520

McCool CS, Li X, Wilhite GP (2009) Flow of a polyacrylamide/chromium acetate system in a long conduit. SPE J 14:54-66

Rousseau D, Chauveteau G, Renard M, Tabary R, Zaitoun A, Mallo P (2005) Rheology and transport in porous media of new water shutoff/conformance control microgels. Paper SPE 93254 presented at the SPE international symposium on oilfield chemistry, Houston; 2-4 February 
Sang Q, Li Y, Yu L, Li Z, Dong M (2014) Enhanced oil recovery by branched-preformed particle gel injection in parallel-sandpack models. Fuel 136:295-306

Seright RS (2001) Gel propagation through fractures SPE production and facilities 16:225-231

Seright R (2003) Washout of Cr (III)-Acetate-HPAM gels from fractures. In: International symposium on oilfield chemistry. Society of Petroleum Engineers

Sun F, Yao Y, Li GJJoPE, Technology P (2019) Slight insights and perspectives of future heavy oil recovery. 1-3

Sydansk R, Xiong Y, Al-Dhafeeri A, Schrader R, Seright R (2004) Characterization of partially formed polymer gels for application to fractured production wells for water-shutoff purposes. In: SPE/ DOE symposium on improved oil recovery. Society of Petroleum Engineers

Tongwa P, Nygaard R, Bai B (2013a) Evaluation of a nanocomposite hydrogel for water shut-off in enhanced oil recovery applications: design, synthesis, and characterization. J Appl Polym Sci 128:787-794
Tongwa P, Nygaard R, Blue A, Bai B (2013b) Evaluation of potential fracture-sealing materials for remediating $\mathrm{CO}_{2}$ leakage pathways during $\mathrm{CO}_{2}$ sequestration. Int $\mathrm{J}$ Greenhouse Gas Control 18:128-138

Wang Y, Seright RS (2006) Correlating gel rheology with behavior during extrusion through fractures. In: SPE/DOE symposium on improved oil recovery. Society of Petroleum Engineers

Wu Y-S, Bai B (2008) Modeling particle gel propagation in porous media. In: SPE annual technical conference and exhibition. Society of Petroleum Engineers

Yu K, Li K, Li Q, Li K, Yang F (2017) A method to calculate reasonable water injection rate for M oilfield. J Pet Explor Prod Technol 7:1003-1010

Zhang H (2014) Preformed particle gel transport through fractures

Publisher's Note Springer Nature remains neutral with regard to jurisdictional claims in published maps and institutional affiliations. 